This is an Open Access article distributed under the terms of the Creative Commons Attribution-Noncommercial License, which permits unrestricted use, distribution, and reproduction in any noncommercial medium, provided the original work is properly cited.

\title{
A MILESTONE TO SPICA EXTRAGALACTIC SURVEYS: THE AKARI NEP SURVEY
}

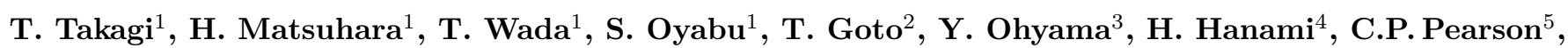
S. Serjeant ${ }^{6}$, M. Negrello ${ }^{6}$, G. White ${ }^{6}$, M. Im ${ }^{7}$, H.M. Lee ${ }^{7}$, M. Malkan ${ }^{8}$, and the AKARI extragalactic team

\author{
${ }^{1}$ Institute of Space and Astronautical Science, Japan Aerospace Exploration Agency, Japan \\ ${ }^{2}$ Institute for Astronomy, University of Hawaii, USA \\ ${ }^{3}$ Academia Sinica, Institute of Astronomy and Astrophysics, Taiwan \\ ${ }^{4}$ Physics section, Faculty of Humanities and Social Sciences, Iwate University, Japan \\ ${ }^{5}$ Rutherford Appleton Laboratory, UK \\ ${ }^{6}$ Department of Physics, The Open University, UK \\ ${ }^{7}$ Department of Physics \& Astronomy, FPRD, Seoul National University, Korea \\ ${ }^{8}$ Department of Physics and Astronomy, UCLA, USA
}

\begin{abstract}
Large area surveys in the infrared wavelengths have revealed a significant evolution of the star formation activity of the universe in the past. The extragalactic survey we have conduced with AKARI towards the north ecliptic pole (NEP) is unique, in terms of a comprehensive wavelength coverage from 2 to 24 micron using all 9 photometric bands of the Infrared Camera (IRC). We demonstrate that this IRC all-band photometry is capable of tracing a steep rise in the flux at the blue side of the polycyclic aromatic hydrocarbon (PAH) 6.2 micron emission feature of infrared luminous galaxies at $z<1$. This allows us to estimate redshifts of mid-IR sources and identify 'ultra-luminous starburst galaxies', based on mid-IR spectral energy distributions (SEDs). SPICA could inherit this unique capability of AKARI and extend the study to typical galaxy populations at $z \sim 2$, i.e. a critical period of galaxy formation.
\end{abstract}

Key words: Galaxies: formation - Galaxies:infrared - Missions: SPICA - Missions: AKARI

\section{INTRODUCTION}

One of the surprising results from Spitzer observations of distant ultraluminous infrared galaxies (defined with $10^{12}<L_{I R}<10^{13} L_{\odot}-$ ULIRGs) is that some ULIRGs have greater PAH emission than local galaxies of similar luminosity (e.g. Rigby et al., 2008). Their mid-infrared spectra resemble that of more than 2 orders of magnitude less luminous starburst galaxies (Valiante et al., 2007; Pope et al., 2008; Farrah et al., 2008). Since these galaxies have been discovered using spectroscopic observations with Spitzer/IRS, it is difficult to assess the statistical properties of these new type of ULIRGs. This is an urgent issue to be resolved.

We are currently undertaking the study of distant infrared galaxies using AKARI's new extragalactic survey towards the north ecliptic pole (NEP), which have comprehensive wavelength coverage at $2-24 \mu \mathrm{m}$, filling the wavelength gap between Spitzer IRAC and MIPS. This survey has unique capability to identify a rough shape of PAH emission feature with photometric observations. Also, AGN identification using mid-infrared properties is more reliable than the case of Spitzer surveys. Here we describe some highlights from the AKARI NEP survey using these capabilities.

\section{The NEP-Deep Survey: Design And Performance}

Owing to a strong visibility constraint of AKARI, large area surveys with pointed (hence deep) observations are possible only around the ecliptic poles. We have chosen the NEP for extragalactic surveys, given the presence of the Large Magellanic Cloud around the south ecliptic pole. The NEP area has been covered by two extragalactic surveys; the NEP-Deep and -Wide survey. This paper describes the NEP-Deep survey. All nine photometric bands in IRC have been used for both surveys to continuously cover $2-24 \mu \mathrm{m}$ wavelength range. Further details of the NEP-Deep survey design can be found in Wada et al. (2008).

We summarize the survey sensitivity in Table 1 . The NEP-Deep survey reached a depth similar to the SWIRE survey with Spitzer (Lonsdale et al., 2004), but covers Spitzer's band gap with 11, 15 and $18 \mu \mathrm{m}$. Deep optical ( $B V R i^{\prime} z^{\prime}$ and $\left.N B 711\right)$ images with the Subaru/Suprimecam (S-cam), reaching the limiting magnitude of $B=28$ and $z^{\prime}=26 \mathrm{AB}$ mag, are available for a part of the NEPDeep field with the area of $27^{\prime} \times 34^{\prime}$, i.e. one field-of-view of S-cam. We have ground-based near-IR $\left(J K_{s}\right)$ images with KPNO-2.1m/FLAMINGOS for the S-cam field (Imai et al., 2007), reaching $K_{s}=19.5$ in Vega magnitude. The ground-based near-IR images have higher spatial resolution (FWHM of $1.08^{\prime \prime}$ ) than that of IRC, and therefore quite useful to examine the source confusion in IRC images.

\section{Number COUNTS}

Complicated emission features of PAHs affect the redshift distribution of flux limited samples from mid-infrared 


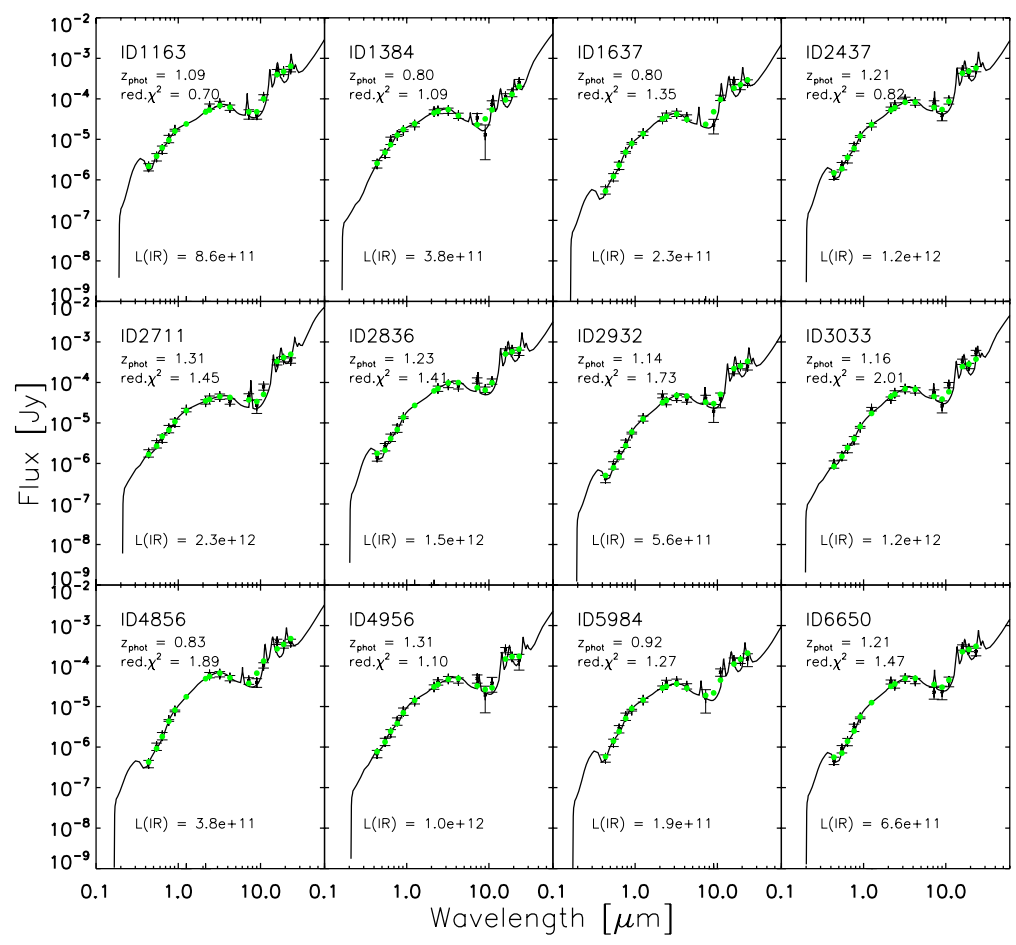

Figure 1. Examples of the SED fitting for PAH-selected galaxies at $z \sim 1$. Data with error bars indicate the observed flux and errors adopted for the SED fitting. Solid circles are filter convolved fluxes of the best fit model.

Table 1. Summary of the NEP-Deep survey

\begin{tabular}{llll}
\hline band & $\begin{array}{l}\text { total area } \\
\left(\operatorname{arcmin}^{2}\right)\end{array}$ & $\begin{array}{l}5 \sigma \text { limit } \\
(\mu \mathrm{Jy})\end{array}$ & $\begin{array}{l}50 \% \text { limit }^{\ddagger} \\
(\mu \mathrm{Jy})\end{array}$ \\
\hline N2 & 2099 & $9.6^{*}$ & $28.8^{*}$ \\
N3 & 2123 & $7.5^{*}$ & $23.5^{*}$ \\
N4 & 2080 & $5.4^{*}$ & $19.7^{*}$ \\
S7 & 2093 & 48.9 & 54.0 \\
S9W & 2078 & 58.5 & 62.6 \\
S11 & 2102 & 70.9 & 80.7 \\
L15 & 2220 & 117.0 & 93.3 \\
L18W & 2226 & 121.4 & 98.8 \\
L24 & 1958 & 275.8 & 209.8 \\
\hline
\end{tabular}

$¥$ Detection limit (sky noise limit).

$\S$ Completeness limit.

* Corrected from the values in Wada et al. (2008), due to misuse of conversion factors.

surveys (Caputi et al., 2006). This has a significant effect on the MIR number counts as well. Furthermore, recent Spitzer/IRS observations of high redshift ULIRGs revealed that mid-infrared properties of high- $z$ ULIRGs are different from local ones; a significant number of ULIRGs show prominent PAH emissions (e.g. Valiante et al., 2007; Farrah et al., 2008; Pope et al., 2008). These PAH-emitting ULIRGs have a PAH feature similar to local starburst galaxies, even though they are over 2 orders of magnitude more luminous. This means that luminosity dependent SED templates of infrared galaxies based on local galaxies may not be applicable for high- $z$ galaxies, even at $z \sim 1$ (see below).

Thus, modeling the number counts can be complicated enough when applied to multiple mid-infrared wavelengths. We have obtained the number counts at 11, 15 and $18 \mu \mathrm{m}$ from the NEP-Deep survey and compared with the model of Pearson (2005) which reproduce both ISO $15 \mu \mathrm{m}$ and Spitzer $24 \mu \mathrm{m}$ number counts. We found that this model overestimate the number of $18 \mu \mathrm{m}$ sources by a factor of 2 (in differential counts). This indicates that, in the midinfrared wavelength range, the multi-band number counts can be useful to constrain the galaxy evolution model, 
even if the difference in wavelength is only $\sim 20 \%$. We have already built a new model to remedy this discrepancy, which will be published elsewhere (Pearson et al. in prep).

\subsection{PAH-SELECTED GALAXIES}

We demonstrate that PAH emission features, specifically a steep rise of flux at the blue side of $\mathrm{PAH} 6.2 \mu \mathrm{m}$ feature, are recognizable in the SED of AKARI/IRC all-band photometry (Figure 1; see also Takagi et al., 2007). Such a steep rise of flux results in very red colors in IRC bands for galaxies at certain redshift ranges. In Figure 2, we show the 11 -to- $7 \mu \mathrm{m}$ flux ratio and the 15 -to- $9 \mu \mathrm{m}$ flux ratio as a function of redshift. These flux ratios have a prominent peak around $z \sim 0.5$ and 1.2 , owing to the PAH emission. At these redshifts, emissions from stellar photosphere dominate the flux at shorter wavelength bands, while PAH emissions dominate at the longer wavelength bands. Thus, these flux ratios can be used as a rough indicator of mass-normalized star formation rate, i.e. specific star formation rate. Since such anomalous flux ratios can be reproduced only by the PAH emission, we select galaxies with these flux ratios greater than 8 as secure candidates of starburst dominated galaxies at $z \gtrsim 0.5$, and call them PAH-selected galaxies. In Figure 3, we show composite optical, near-infrared and mid-infrared color images of three PAH-selected galaxies at $z \sim 0.5$, showing red colors in the $7,9,11 \mu \mathrm{m}$ composite image.

\subsection{PAH-TO-TOTAL IR LUMINOSITY RELATION}

Spitzer spectroscopic observations have established the relation between PAH luminosity and total infrared luminosity for starburst galaxies (Brandl et al., 2006; Houck et al., 2007; Farrah et al., 2007). In Figure 4, we show the PAH $7.7 \mu \mathrm{m}$ peak luminosity from Spitzer IRS observations (compiled in Weedman \& Houck, 2008) against the total infrared luminosity. The local sample $(z<0.2)$ from Spitzer IRS observations is indicated by crosses. A straight line indicates the correlation $\log L_{I R}=\log \left[\nu L_{\nu}(7.7 \mu \mathrm{m})\right]+$ 0.78 taken from Houck et al. (2007). At the high luminosity end, $L_{I R} \gtrsim 10^{12} L_{\odot}$, it is evident that the $7.7 \mu \mathrm{m}$ luminosity saturates, probably because of the AGN contribution to the infrared luminosity and/or the absorption of $7.7 \mu \mathrm{m}$ flux by dust.

Thanks to a good wavelength sampling of the IRC bands, we can estimate the rest-frame $8 \mu \mathrm{m}$ luminosity with almost no $K$-correction. Using this capability, we estimate the $7.7 \mu \mathrm{m}$ peak luminosity from our photometric observations. The rest-frame $8 \mu \mathrm{m}$ luminosity is converted to the $7.7 \mu \mathrm{m}$ peak luminosity with a correction factor of 2.0, considering the effect of filter convolutions.

We here use the sample of IRC all-band-detected sources in the Subaru/Suprime-cam field; i.e. sources detected in all 9 IRC bands. A StarBUrst SED model with Radiative
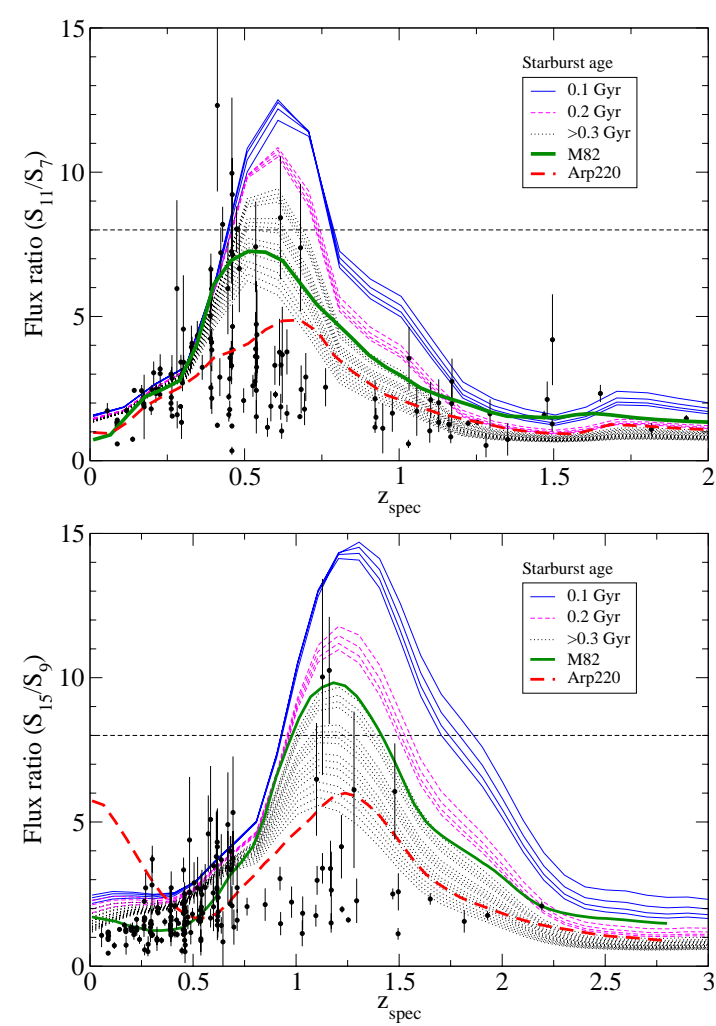

Figure 2. Flux ratio of 11 to $7 \mu m$ (left) and 15 to $9 \mu m$ (right) as a function of redshift. Expected flux ratios from Arp220 and M82 are depicted as thick dashed and solid lines, respectively. A starburst SED model of Takagi et al. (2003) is used for other lines. Horizontal lines indicate the flux ratio of 8 , a selection criterion of PAH-selected galaxies. All-band-detected sources with spectroscopic redshift are indicated with solid circles.

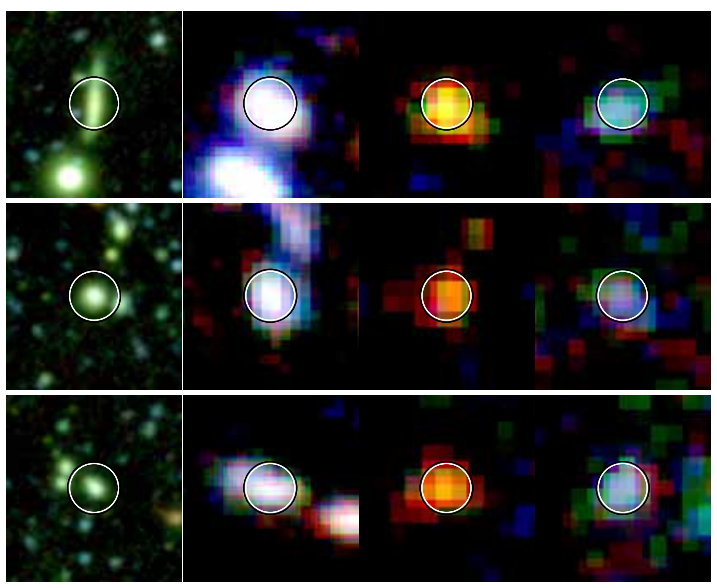

Figure 3. Composite color images of PAH-selected galaxies with Subaru/Suprime-cam $B / R / z^{\prime}$, IRC $N 2 / N 3 / N 4$, $S 7 / S 9 W / S 11$, and $L 15 / L 18 W / L 24$ bands (from left to right). The radius of circles is 4 arcsec. 


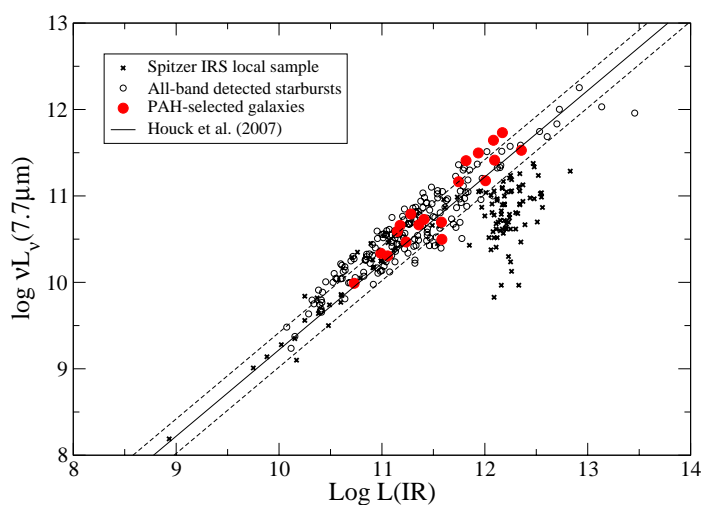

Figure 4. PAH 7.7 $\mu$ m peak luminosity versus total infrared luminosity. Crosses indicate spectroscopic sample at $z<0.2$ taken from Weedman 8 Houck (2008) and references therein. Open and solid circles are for IRC all-band-detected sources and PAH-selected galaxies, respectively.

Transfer (SBURT; Takagi et al., 2003) is applied for this sample, in order to estimate redshift and total infrared luminosity. We used $B V R i z^{\prime} J K$ and IRC photometry for the SED fitting with the same method in Takagi et al. (2007). Examples of the SED fitting results is shown in Figure 1. We confirmed that the estimated total infrared luminosity is reliable within $\sim 50 \%$ by comparing predicted radio fluxes to observations of a subsample.

Figure 4 shows the resulting relation between PAH $7.7 \mu \mathrm{m}$ and total infrared luminosity of IRC all-band-detected sources. Large solid circles indicate PAH-selected galaxies, for which luminosity estimations are most reliable. ULIRGs in our sample lie mostly at $z \sim 1$, and do not show saturation of $7.7 \mu \mathrm{m}$ luminosity. This indicates that even at $z \sim 1$ mid-infrared characteristics of ULIRGs are different from local ones and starburst can be more luminous at high redshifts. Weedman \& Houck (2008) derive the same conclusion by compiling various Spitzer IRS spectroscopic samples. However, it is difficult to derive the number density of most luminous starburst galaxies at high redshifts from such non-uniform spectroscopic samples. Since we can identify luminous starburst galaxies from mid-infrared SED, i.e. photometric observations, thanks to comprehensive wavelength coverage, the NEPDeep survey has a unique capability of constraining the number density of most luminous starburst galaxies.

\section{SUMmary}

We have successfully conducted the largest extragalactic survey with AKARI/IRC pointed observations, which targets the NEP region with the area of $0.38 \mathrm{deg}^{2}$ (NEPDeep). We have achieved a similar depth with the Spitzer SWIRE survey, but with additional mid-infrared bands filling the Spitzer/IRAC and MIPS wavelength gap between 8 and $24 \mu \mathrm{m}$. Unique points of this IRC all-band photometric survey is as follows. A comprehensive wave- length coverage allows us to identify galaxies whose midinfrared emission is dominated by PAHs with photometric observations. Such galaxies, PAH-selected galaxies, can be identified with single IRC colors, such as $S 7-S 11$ and $S 9 W-L 15$. They include most luminous starburst galaxies at $z \sim 1$. We estimated the PAH $7.7 \mu \mathrm{m}$ peak luminosity from the IRC photometry and compared with the total infrared luminosity obtained with a starburst SED model. While local ULIRGs have suppressed PAH luminosity when compared with less luminous starburst galaxies, we find no such suppression for PAH-selected galaxies at $z \sim 1$. This indicates that mid-infrared properties of distant ULIRGs are different from local ones, even at $z \sim 1$.

\section{ACKNOWLEDGEMENTS}

We would like to thank all AKARI team members for their support on this project. This research is based on observations with AKARI, a JAXA project with the participation of ESA.

\section{REFERENCES}

Brandl, B. R., et al. 2006, ApJ, 653, 1129

Caputi, K. I., et al. 2006, ApJ, 637, 727

Farrah, D., et al. 2007, ApJ, 667, 149

—. 2008, ApJ, 677, 957

Houck, J. R., Weedman, D. W., Le Floc'h, E., \& Hao, L. 2007, ApJ, 671, 323

Imai, K., Matsuhara, H., Oyabu, S., Wada, T., Takagi, T., Fujishiro, N., Hanami, H., \& Pearson, C. P. 2007, AJ, 133, 2418

Lonsdale, C., et al. 2004, ApJS, 154, 54

Pearson, C. 2005, MNRAS, 358, 1417

Pope, A., et al. 2008, ApJ, 675, 1171

Rigby, J. R., et al. 2008, ApJ, 675, 262

Takagi, T., Arimoto, N., \& Hanami, H. 2003, MNRAS, 340,813

Takagi, T., et al. 2007, PASJ, 59, 557

Valiante, E., Lutz, D., Sturm, E., Genzel, R., Tacconi, L. J., Lehnert, M. D., \& Baker, A. J. 2007, ApJ, 660, 1060

Wada, T., et al. 2008, PASJ, 60, 517

Weedman, D. W., \& Houck, J. R. 2008, ApJ, 686, 127 